\title{
A Wireless Powered Feedback Protocol for Opportunistic Beamforming using Rectenna Arrays
}

\author{
Constantinos Psomas, Member, IEEE, and Ioannis Krikidis, Senior Member, IEEE
}

\begin{abstract}
This paper proposes an opportunistic beamforming (OBF) protocol with limited feedback in the context of wireless powered communication networks. The terminals are randomly deployed around an access point (AP) and are equipped with multiple antennas as well as a rectenna array to harvest energy. In the OBF scheme, the AP acquires channel related feedback from the network's terminals and allocates the orthonormal beams to the users with the best link. We consider the case, where the feedback is solely powered by energy harvested from electromagnetic radiation. Specifically, each terminal adjusts the length of its feedback based on the amount of harvested energy; when the energy is not sufficient to feed back at least one bit, the terminal is considered to be in outage. We study two fundamental rectenna architectures, the direct-current combiner and the radio-frequency combiner (coherent and non-coherent), as well as a hybrid architecture of these two. Furthermore, to alleviate the doubly near-far problem, we consider a scheme where a terminal adapts the number of rectenna elements according to its distance from the origin. By using higher order statistics and stochastic geometry, the beam outage probability is derived in closed form for all considered architectures and scenarios.
\end{abstract}

Index Terms-Opportunistic beamforming, wireless power transfer, antenna array, stochastic geometry.

\section{INTRODUCTION}

Knowledge of channel state information (CSI) in wireless communication networks is crucial for enhancing the information transfer between a receiver and a transmitter. A well-known approach in obtaining CSI at the transmitter is the employment of a feedback mechanism. In this case, the receiver feeds back information to the transmitter regarding its channel and, in turn, the transmitter adapts its operation accordingly based on the acquired knowledge [2]. The study of feedback in communication systems has a rich history, dating back to the time of Shannon [3], and can also be found in recent IEEE standards [4]. Among the numerous communication techniques that exploit the use of feedback, opportunistic communication is characterized for its simplicity as well as efficiency in improving the network's performance. According to this technique, in a point-to-point scenario, the transmitter increases or reduces its power based on whether the

C. Psomas and I. Krikidis are with the KIOS Research and Innovation Center of Excellence, Department of Electrical and Computer Engineering, University of Cyprus, Nicosia 1678, Cyprus (e-mail: \{psomas, krikidis\}@ucy.ac.cy). Preliminary results of this work were presented at the IEEE International Conference on Communications, Kuala Lumpur, Malaysia, 2016 [1].

This work was supported in part by the Research Promotion Foundation, Cyprus under the project COM-MED with pr. no. KOINA/ERANETMED/1114/03, by the European Unions Horizon 2020 research and innovation programme under grant agreement No 739551 (KIOS CoE) and by the Government of the Republic of Cyprus through the Directorate General for European Programmes, Coordination and Development. channel is weak or strong. On the other hand, in a large-scale scenario, the access point (AP) schedules its transmissions to the users with the best channels; the network, in this case, experiences multiuser diversity since with high probability one of the users will have a strong channel [2]. Thus, by having knowledge of the network's channel conditions, the transmitter can use its resources more efficiently and serve the user with the best channel to maximize the system's throughput.

Opportunistic beamforming $(\mathrm{OBF})$ is an opportunistic communication technique implemented in a multiple-input multiple-output (MIMO) scenario [5]. OBF is a well-known low-complexity beamforming scheme which exploits channel randomness and multiuser diversity to obtain full multiplexing gain. In the OBF scheme, the AP assigns specific orthonormal beams to the terminals based on the acquired feedback regarding the achieved beam signal-to-interference-plus-noise ratio (SINR). One of the main advantages of OBF is that it increases the sum-rate capacity in the case of full CSI (in the form of SINRs) and a large number of terminals [6]. Many works exist which study the OBF scheme under various assumptions and scenarios, e.g. [6]-[9]. In [6], the authors consider MIMO broadcast channels with partial CSI and show that for large number of users $n$ with $M$ antennas, the throughput scales as $M \log \log n$. Similarly, the authors in [7] study a MIMO broadcast channel, but consider the asymptotic case $n \rightarrow \infty$ and provide the fundamental feedback limits to achieve the sum-rate capacity with full CSI; they show that for low signalto-noise ratio (SNR) values, the OBF scheme is optimal. The work in [8] studies the sum-rate performance of opportunistic scheduling at the downlink and show that significant gains can be achieved when the users employ low-complexity linear precoding schemes. Furthermore, a large-scale approach is considered in [9] by also taking into account the path-loss effects; analytical expressions for the beam outage probability and ergodic aggregate data rate of an AP are derived. Despite the performance benefits associated with feedback, its implementation significantly increases the complexity of a system. To overcome this, limited feedback can be employed. This can reduce the system's complexity and it is sufficient to achieve near optimal system performance [10], [11]. As already discussed above, OBF can be implemented with partial CSI, i.e. limited feedback for the achieved SINRs [6], [7]. Indeed, the authors in [12], have shown that the optimal scaling law for the sum-rate can be achieved with just one bit of feedback based on a certain pre-assignment of the beams. Moreover, the work in [13] proposes a combined approach where just one bit of feedback is used for CSI whereas the remaining bits are used to choose a specific beamforming vector; it is shown that this method achieves the same capacity 
growth as the single-beam full CSI OBF.

Now, even though the complexity can be reduced, the OBF scheme still requires a continuous transmission of feedback from the terminals which is very demanding on their energy and bandwidth resources. A promising solution to overcome these energy constraints is wireless power transfer (WPT), i.e. harvesting energy from electromagnetic radiation, which has recently emerged as a practical technique to increase the energy efficiency of wireless networks [14]. Three fundamental architectures exist for wireless powered networks. The first, WPT, refers to energy transfer solely over the downlink [15]; the second, simultaneous wireless information and power transfer (SWIPT), refers to the transmission of both information and energy transfer by the AP over the same radio frequency (RF) signal [16]. Finally, the third, wireless powered communication networks (WPCNs), refers to networks where the terminals harvest energy from RF signals, transmitted by a dedicated power beacon (PB) and use it to power their uplink transmissions [17]; note that the AP and the PB operate in different frequency bands to avoid interference. The harvesting operation is implemented with a rectifying-antenna (rectenna) which is a diode-based circuit that converts the RF signals to direct-current (DC) voltage [18]. Due to the nature of electromagnetic radiation, the harvesting efficiency highly depends on parameters at the transmitter's side such as the transmit power and the employed transmission techniques, e.g. energy beamforming [19]. On the other hand, at the receiver's side, several rectennas can be combined and their output amalgamated in order to increase the level of harvested energy [15]. This amalgamation can either be done at the DC domain, where the total power is produced after each rectification circuit outputs a DC current, or at the RF domain, where the signals are first combined and then passed to a single rectification circuit; these two architectures can also be combined [20], [21].

In this paper, we consider a multi-antenna WPCN implementing the OBF scheme with limited feedback whose length depends on the amount of harvested energy. Each terminal employs a rectenna array and harvests energy from the RF signals transmitted by the PB in order to power its feedback operation. Given the sensitivity of the rectenna to signal propagation, we study this scenario from a largescale point-of-view by also taking into account the path-loss effects. Apart from [9] and [22], most works ignore spatial randomness and path-loss effects on the OBF performance. Moreover, [9] studies the OBF scheme in the case where the terminals return full CSI feedback. On the other hand, the work in [22], studies a WPCN-based OBF network where the terminals harvest RF signals but return just one bit of feedback. Therefore, motivated by the above, this paper's contributions are as follows:

- We consider a variable length limited feedback scheme, where each terminal returns a finite number of bits, subject to the harvested energy being sufficient to fulfil that transmission. In this way, we accommodate the doubly near-far problem in the sense that a terminal closer to the AP will be able to return a feedback of larger length than a terminal located further away.
TABLE I: Notation summary

\begin{tabular}{|l|l|}
\hline Notation & Description \\
\hline$\Phi$ & Homogeneous PPP \\
\hline$\lambda$ & Density of PPP $\Phi$ \\
\hline $\mathcal{B}$ & Coverage area \\
\hline$\rho$ & Coverage area radius \\
\hline$\xi$ & Exclusion zone radius \\
\hline$\alpha$ & Path-loss exponent \\
\hline$d_{i}$ & Distance to $i$-th terminal \\
\hline$P_{t}$ & AP's transmit power \\
\hline$P_{h}$ & PB's transmit power \\
\hline$\zeta$ & Combiner's efficiency \\
\hline$M$ & Number of AP antennas \\
\hline$K$ & Number of terminal antennas \\
\hline$N$ & Number of data decoding antennas \\
\hline$L$ & Number of energy harvesting antennas \\
\hline$\tau$ & Beam SINR threshold \\
\hline$\gamma_{i, j, l}$ & $i$-th terminal, $j$-th antenna, $l$-th beam SINR \\
\hline$\gamma_{i, l}$ & $i$-th terminal, $l$-th beam combined SINR \\
\hline$\gamma_{l}^{*}$ & Maximum $l$-th beam SINR \\
\hline
\end{tabular}

- Two fundamental rectenna array architectures are studied, namely the DC and RF combiners, as well as a hybrid architecture of the two. Furthermore, the case where the signals at the RF architecture are received coherently is also considered. A comparison of these architectures is presented in terms of their harvesting performance.

- We assume the terminals employ low-complexity antenna selection schemes to operate their feedback mechanisms based on three antenna assignment scenarios. Firstly, we consider a static scenario where the number of antennas used for decoding and harvesting is decided a-priori. Secondly, we consider a dynamic assignment scenario according to a distance-based protocol where the terminals further away from the AP, assign more antennas for energy harvesting. In this way, this scenario attempts to alleviate the doubly near-far problem. Finally, we consider a network-wide optimal scenario which maximizes the network's performance. By using higher order statistics and stochastic geometry tools, analytical expressions for the beam outage probability are derived for the considered scenarios.

Although this work considers OBF, the ideas proposed throughout the paper regarding wirelessly powered feedback are general and can be applied in any wireless communication system.

The rest of the paper is organized as follows: Section II presents the network model, the main assumptions regarding both information and power transfer as well as a detailed description of the considered rectenna architectures. Section III presents the main steps of the proposed wireless powered OBF protocol. Next, in Section IV, analytical expressions for the harvesting performance of the rectenna architectures are derived and Section $\mathrm{V}$ provides mathematical analysis of the beam outage probability for the considered scenarios. In Section VI, the numerical results are presented and finally, the conclusions of this work are given in Section VII.

Notation: Lower case boldface letters denote vectors; $N_{\mathcal{Z}}(A)$ denotes the number of terminals in the area $A$ for a Poisson point process $\mathcal{Z} ; \Re\{z\}$ denotes the real part of $z$ and $\jmath=\sqrt{-1}$ denotes the imaginary unit; $\mathbb{P}\{X\}$ denotes 


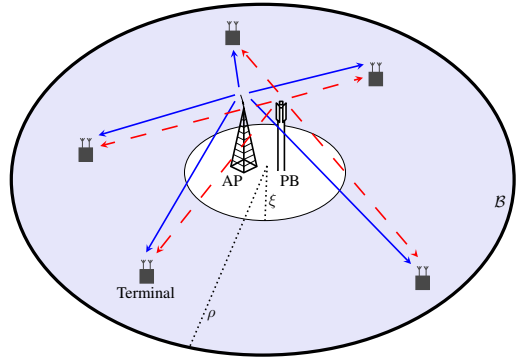

Fig. 1: The considered system model; solid and dashed lines depict communication and power links, respectively, the shaded and unshaded regions illustrate the coverage and exclusion zones, respectively.

the probability of the event $X$ and $\mathbb{E}\{X\}$ represents the expected value of $X ; n ! !=n(n-2)(n-4) \cdots$ denotes the double factorial of the non-negative integer $n ; \Gamma(\cdot)$ and $\Gamma(\cdot, \cdot)$ denote the complete and upper incomplete Gamma functions respectively [23]. Finally, the main mathematical notation used in the paper is summarized in Table I.

\section{SYSTEM MODEL}

\section{A. Network and channel model}

We consider a WPCN with multiple randomly deployed terminals in a single cell. The terminals are spatially distributed according to a homogeneous Poisson point process (PPP) $\Phi$ in the Euclidean plane, with density $\lambda$ [24]. The coverage area, denoted by $\mathcal{B}$, is modeled as a disc of radius $\rho$. An AP and a $\mathrm{PB}$ are co-located at the origin of the disc with a circular exclusion zone of radius $\xi$ around them [18]; the PB could be integrated into the AP or it could be deployed separately. Due to the PB's high radiation power, the exclusion zone acts as a prohibited area for safety reasons and guarantees that all terminals are in the far-field of the PB; the length of the radius $\xi$ depends on the frequency and power density $\left(\mathrm{W} / \mathrm{m}^{2}\right)$ of the transmitter [25]. The PB operates at a different frequency band to the AP in order to avoid interfering with the communication links [18]. Fig. 1 schematically presents the considered system topology. The AP is equipped with $M$ antennas, while the $\mathrm{PB}$ has a single transmit antenna. The terminals employ $K$ antennas connected to one of two different combiner circuits: one for data decoding with $N$ antenna elements and the other for energy harvesting with $L$ antenna elements, where $K=N+L$. All antennas are considered to be omnidirectional. Each terminal harvests energy through its rectenna array configuration of $L$ antenna elements, from the PB's transmitted signals [21]. Depending on the energy harvesting performance of the rectenna array, a feedback of $b$ bits is returned to the AP; $b$ is a discrete random variable that takes an integer value between 1 and $M$. We consider a batteryless architecture so any energy harvested is not stored but is used immediately to potentially operate the terminal's feedback mechanism [17].

We assume that all downlink wireless links suffer from both small-scale block fading and large-scale path-loss effects. The fading is considered to be Rayleigh distributed so the power of the channel fading is an exponential random variable with unit variance. We denote by $h_{k, i, j}$ the channel coefficient for the link between the $k$-th transmit antenna of the AP and the $j$-th receive antenna of the $i$-th terminal. The channel coefficient for the link between the PB and the $j$-th rectenna of the $i$-th terminal is denoted by $g_{i, j} \equiv\left|g_{i, j}\right| \exp \left(\jmath \theta_{i, j}\right)$. Moreover, all wireless links exhibit additive white Gaussian noise (AWGN) with variance $\sigma^{2}$; we denote by $n_{i, j}$ the AWGN at the $j$-th receive antenna of the $i$-th terminal. The pathloss model assumes that the received power is proportional to $d_{i}^{-\alpha}$ where $d_{i}$ is the Euclidean distance from the origin to the $i$-th terminal and $\alpha>2$ is the path-loss exponent. Hence, the probability density function of the distance $d_{i}$ is given by $f_{d}(x)=\frac{1}{\pi\left(\rho^{2}-\xi^{2}\right)}$. For simplicity, the feedback channel at the uplink is assumed to suffer only from path-loss effects, i.e. channel fading is ignored [26]. Therefore, a single antenna is randomly selected for the transmission and reception of the feedback channel.

\section{B. Information and power transfer}

The AP employs the OBF scheme to serve the selected terminals. It transmits $M$ information streams, one for each beam, by generating $M$ isotropic distributed random orthonormal vectors $\left\{\boldsymbol{u}_{1}, \ldots, \boldsymbol{u}_{M}\right\}$ with $\boldsymbol{u}_{m} \in \mathbb{C}^{M \times 1}, 1 \leq m \leq M$. By omitting time index and carriers, the baseband-equivalent transmitted signal is given by

$$
\boldsymbol{v}=\sum_{m=1}^{M} \boldsymbol{u}_{m} s_{m},
$$

where $\mathbb{E}\left\{\|\boldsymbol{v}\|^{2}\right\}=M$ and $s_{m}$ is the $m$-th transmitted symbol with $\mathbb{E}\left\{\left|s_{m}\right|^{2}\right\}=1$ for all $m=1, \ldots, M$. Then, the signal received at the $j$-th antenna of the $i$-th terminal is given by

$$
r_{i, j}=\sqrt{P_{t} d_{i}^{-\alpha}} \boldsymbol{h}_{i, j}^{T} \boldsymbol{v}+n_{i, j},
$$

where $\boldsymbol{h}_{i, j}^{T}=\left[h_{1, i, j}, \ldots, h_{M, i, j}\right]$ and $P_{t}$ denotes the AP's transmit power. We assume the terminals obtain knowledge of the beamforming vectors through training [6]. Therefore, the SINR for the $l$-th beam at the $j$-th receive antenna of the $i$-th terminal can be written as

$$
\gamma_{i, j, l}=\frac{P_{t}\left|\boldsymbol{h}_{i, j}^{T} \boldsymbol{u}_{l}\right|^{2}}{\sigma^{2} d_{i}^{\alpha}+P_{t} \sum_{m \neq l}^{M}\left|\boldsymbol{h}_{i, j}^{T} \boldsymbol{u}_{m}\right|^{2}} .
$$

In order to increase the energy efficiency and decrease the implementation complexity, we assume that each terminal employs a generalized selection combining (GSC) scheme [27]. Hence, by assuming that $\gamma_{i, 1, l}>\gamma_{i, 2, l}>\cdots>\gamma_{i, N, l}$, the achieved SINR for the $l$-th beam at the $i$-th terminal is given by

$$
\gamma_{i, l}=\sum_{j=1}^{N_{c}} \gamma_{i, j, l},
$$

where $N_{c} \leq N$ is the number of branches combined.

Each terminal attempts to harvest energy from the PB's transmitted signal, for the sole purpose of operating its feedback scheme. In this case, the transmitted RF signal from the $\mathrm{PB}$ is given by

$$
s(t)=\sqrt{2 P_{h}} \Re\left\{x(t) \exp \left(\jmath 2 \pi f_{c} t\right)\right\}
$$




$$
=\sqrt{2 P_{h}} \Re\left\{\exp \left(\jmath\left[2 \pi f_{c} t+\arg x(t)\right]\right)\right\},
$$

where $P_{h}=\mathbb{E}\left\{s^{2}(t)\right\}$ is the PB's transmit power, $f_{c}$ denotes the carrier frequency, and $x(t)$ is a modulated energy signal with $|x(t)|^{2}=1$. Thus, the received signal at the $j$-th antenna of the $i$-th terminal is given by

$$
\begin{aligned}
y_{i, j}(t)= & \sqrt{2 P_{h} d_{i}^{-\alpha}}\left|g_{i, j}(t)\right| \\
& \times \Re\left\{\exp \left(\jmath\left[2 \pi f_{c} t+\arg x(t)+\theta_{i, j}(t)\right]\right)\right\} \\
= & \sqrt{2 P_{h} d_{i}^{-\alpha}}\left|g_{i, j}(t)\right| \cos \left(2 \pi f_{c} t+\arg x(t)+\theta_{i, j}(t)\right),
\end{aligned}
$$

where $\left|g_{i, j}(t)\right|$ is a Rayleigh random variable with unit scale parameter. The received signal at each antenna is then converted to a DC output with the use of a rectifier and any $\mathrm{RF}$ energy harvesting from the AWGN is considered to be negligible and thus it is ignored [16], [17]. A rectifier is a basic circuit, usually consisting of a diode (e.g. a Schottky diode) and a passive low pass filter (LPF) [28]. This circuit essentially defines the conversion efficiency from RF to DC; in fact, the efficiency is mainly determined by the diode. The output current of the diode for the $j$-th antenna element of the $i$-th terminal is given by

$$
I_{i, j}(t)=I_{s}\left(\exp \left(\frac{y_{i, j}(t)}{\mu V_{T}}\right)-1\right) \stackrel{(a)}{=} I_{s} \sum_{k=1}^{\infty} \frac{1}{k !}\left(\frac{y_{i, j}(t)}{\mu V_{T}}\right)^{k},
$$

where $I_{s}$ denotes the reverse saturation current of the diode, $\mu \in\left[\begin{array}{ll}1 & 2\end{array}\right]$ is an ideality factor which is a function of the operating conditions and physical contractions, and $V_{T}$ is the thermal voltage; $(a)$ follows from the Taylor series expansion of an exponential function [28].

\section{Rectenna array architectures}

Each terminal is equipped with a rectenna array consisting of $L$ antenna elements in order to boost the rectification process and increase its efficiency. The interconnection of these elements can be performed either in the DC domain, the RF domain or a hybrid combination of the two [20], [21]; these are illustrated in Fig. 2. We consider a hybrid DC/RF combiner architecture (see Fig. 2a), implementing $L_{r} \leq L$ elements over $\ell$ sub-arrays of the RF combiner such that $L_{r}=\sum_{k=1}^{\ell} L_{r_{k}}$, where $L_{r_{k}}$ is the number of antenna elements of the $k$-th RF combiner, and a sub-array of $L_{d}=L-L_{r}$ elements directly connected to the DC combiner. As such, each RF combiner merges its antenna inputs in the RF domain and all $\ell+L_{d}$ outputs are then combined in the DC domain. Each antenna element of the DC combiner has its own rectification circuit and is not affected by the remaining elements. Then, the signal at the $j$-th antenna of the $i$-th terminal is given by (6), $1 \leq j \leq L_{d}$. On the other hand, the RF combiner merges the antenna inputs in the RF domain and thus requires only a single rectification circuit. In this case, the signals can be combined in two ways, namely coherently and non-coherently.

In the coherent case, the rectenna array aligns the phases of the incoming signals so that they are combined co-phased; several implementations of this technique have been proposed

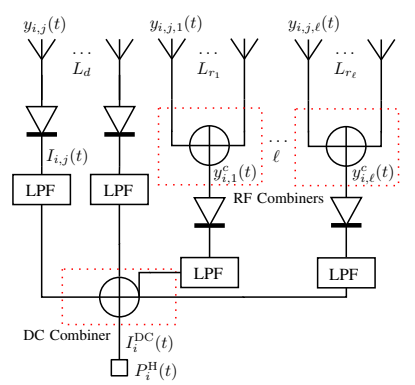

(a)

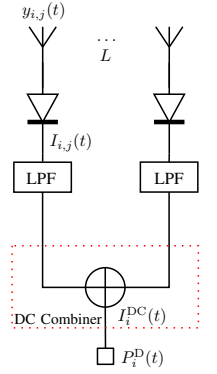

(b)

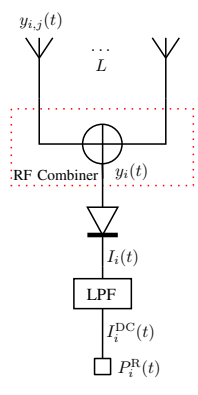

(c)
Fig. 2: Rectenna array architectures for WPT; (a) Hybrid combiner, (b) DC combiner, (c) RF combiner.

[29], [30]. Specifically, a phase shifter circuit can be employed to form a beam by continuously shifting the phase of the incoming signal at each antenna. This technique may add some extra cost to the combiner due to the employment of the phase alignment circuit, but it does not necessarily add any further complexity or power consumption since it can be implemented with passive elements [29], [30]. In this case, the combined signal $y_{i, k}^{c}(t)$ at the $k$-th RF combiner of the $i$-th terminal is given by

$$
\begin{aligned}
& y_{i, k}^{c}(t)=\sum_{j=1}^{L_{r_{k}}} y_{i, j, k}(t) \\
& =\sqrt{2 P_{h} d_{i}^{-\alpha}} \\
& \quad \times \Re\left\{\exp \left(\jmath\left[2 \pi f_{c} t+\arg x(t)+\theta_{i}(t)\right]\right) \sum_{j=1}^{L_{r_{k}}}\left|g_{i, j, k}(t)\right|\right\} \\
& =\sqrt{2 P_{h} d_{i}^{-\alpha}} \cos \left(2 \pi f_{c} t+\arg x(t)+\theta_{i}(t)\right) \sum_{j=1}^{L_{r_{k}}}\left|g_{i, j, k}(t)\right|,
\end{aligned}
$$

where $y_{i, j, k}(t)$ is the received signal at the $k$-th RF combiner's $j$-th antenna of the $i$-th terminal and $\left|g_{i, j, k}(t)\right|$ is a Rayleigh random variable with unit scale parameter. In the non-coherent case, the signals are combined non-coherently and so no $\mathrm{RF}$ alignment is required. Therefore, the non-coherent RF combiner is characterized by its simplicity. Here, the combined signal is

$$
\begin{aligned}
& y_{i, k}^{c}(t)=\sum_{j=1}^{L_{r_{k}}} y_{i, j, k}(t) \\
& =\sqrt{2 P_{h} d_{i}^{-\alpha}} \\
& \quad \times \Re\left\{\exp \left(\jmath\left[2 \pi f_{c} t+\arg x(t)\right]\right) \sum_{j=1}^{L_{r_{k}}}\left|g_{i, j, k}(t)\right| \exp \left(\jmath \theta_{i, j, k}(t)\right)\right\} \\
& =\sqrt{2 P_{h} d_{i}^{-\alpha}}\left|c_{i, k}(t)\right| \cos \left(2 \pi f_{c} t+\arg x(t)+\theta_{i, k}(t)\right),
\end{aligned}
$$

where $\sum_{j=1}^{L_{r_{k}}}\left|g_{i, j, k}(t)\right| \exp \left(\jmath \theta_{i, j, k}(t)\right)=\left|c_{i, k}(t)\right| \exp \left(\jmath \theta_{i, k}(t)\right)$ is a circularly-symmetric complex Gaussian random variable with zero mean and variance $L_{r_{k}}$. 
The output current from each diode is processed by the LPF which produces a relatively smooth DC current. We consider a linear model for the rectifier [28], so the output DC current is given by

$$
I_{i}^{\mathrm{DC}}(t)=\frac{I_{s} P_{h} d_{i}^{-\alpha}}{\left(\mu V_{T}\right)^{2}}\left(\sum_{j=1}^{L_{d}}\left|g_{i, j}(t)\right|^{2}+\sum_{k=1}^{\ell} \omega_{k}(t)\right),
$$

where $\left|g_{i, j}(t)\right|^{2}$ is the output of the $j$-th rectenna directly connected to the DC combiner, and $\omega_{k}(t)$ is the output of the $k$-th $\mathrm{RF}$ combiner given by

$$
\omega_{k}(t)= \begin{cases}\left(\sum_{j=1}^{L_{r_{k}}}\left|g_{i, j, k}(t)\right|\right)^{2} & \text { coherent RF combiner, } \\ \left|c_{i, k}(t)\right|^{2} & \text { non-coherent RF combiner, }\end{cases}
$$

where $\left|g_{i, j, k}(t)\right|$ is the input from the $j$-th antenna element to the $k$-th coherent RF combiner and $\left|c_{i, k}(t)\right|^{2}$ is the output of the $k$-th non-coherent RF combiner. Then, the total harvested DC power is a linear function of $I_{i}^{\mathrm{DC}}(t)$, that is,

$$
P_{i}(t)=\zeta I_{i}^{\mathrm{DC}}(t)
$$

where $\zeta$ is the the combiner's conversion efficiency which is defined as the ratio of the harvested DC power to the received signal power [21]. Note that the total harvested DC power from a strictly DC combiner (Fig. 2b) is given by simply setting $\ell=0$ to (10) or when $\ell>0$ and $L_{r_{i}}=1$, for all $i$; similarly, for a strictly RF combiner (Fig. 2c), it is given by setting $L_{d}=0$ to (10).

\section{WIRELESS POWERED OBF PROTOCOL}

The main aim of the OBF scheme is to maximize the sumrate by assigning the $M$ beams to the $M$ "best" terminals. In the conventional scheme, each terminal returns a feedback to the AP consisting of the achieved SINR from each beam. Then, the AP assigns each beam to the terminal with the highest SINR for that specific beam. However, this approach has significant demands in terms of system resources, such as bandwidth and energy, since it requires the transmission of a large amount of information. The proposed wireless powered OBF scheme considers terminals that harvest energy from RF signals in order to power their feedback transmission ${ }^{1}$. The transmitted feedback is of finite length and it is entirely determined by the harvested energy. Each terminal employs a harvest-then-transmit protocol and so the energy harvested is immediately used for the feedback channel [17]. Consequently, the proposed protocol increases the energy efficiency of the terminals and in turn increases the efficiency of the considered network. In what follows, we provide a detailed step-by-step description of the proposed protocol:

1. A random ordered pre-assignment of the $M$ beams for each terminal is performed, which ensures that each beam is considered in the network with equal probability. All assignments are known to the AP and each terminal

\footnotetext{
${ }^{1} \mathrm{We}$ assume low-powered terminals where the energy consumption to operate the implemented circuit is negligible and is dominated by the transmit power [31].
}

has knowledge of its own ordered assignment which is acquired through an initialization process of the system.

2. The AP broadcasts $M$ beamforming vectors and the PB transmits RF signals to the terminals over different frequency bands.

3. Each terminal harvests energy through its rectenna array configuration. If the harvested energy is sufficient to return $b \geq 1$ bits of feedback, the terminal measures the SINR for the first $b$ beams in its ordered assignment. Otherwise, the terminal remains idle.

4. Each active terminal transmits $b$ bits of feedback back to the AP, $1 \leq b \leq M$. The feedback is of binary form and consists of one bit per beam where the $i$-th bit describes whether or not the $i$-th measured SINR is above a preassigned threshold $\tau$.

5. The AP evaluates the received feedback and randomly assigns each beam to a terminal which returned a positive feedback for that beam. If all feedback for a specific beam is negative, the assignment is done randomly.

The one-bit-per-beam approach is considered for the following reasons ${ }^{2}$. Firstly, a certain pre-assignment of the beams can achieve the optimal scaling law of the sum-rate with just one bit of feedback [12]. Secondly, the small efficiency of the WPT process and the associated doubly near-far problem makes the one bit feedback channel ideal in this context [22]. Of course, the efficiency of the WPT process highly depends on the employed rectenna array architecture. In the next section, we derive the probability of a terminal returning a feedback of finite length for each rectenna array architecture.

\section{FEEDBACK LENGTH \& IDLE PROBABILITY}

A terminal harvests energy from the PB's transmitted signals and attempts to return a feedback of length up to $M$ bits. In the case where the harvested energy is sufficient to return at least one bit, the terminal becomes active, otherwise it remains idle. Therefore, for the $i$-th terminal to transmit a feedback of random number of $b$ bits, $1 \leq b \leq M$, the Shannon capacity of the uplink between the terminal and the AP should be greater or equal to $b$ bits per channel use, that is,

$$
C_{\mathcal{Q}}=\log _{2}\left(1+\frac{P_{i}^{\mathcal{Q}}(t)}{d_{i}^{\alpha} \sigma^{2}}\right) \geq b \Rightarrow P_{i}^{\mathcal{Q}}(t) \geq \phi(b) d_{i}^{\alpha} \sigma^{2}
$$

where $\phi(b) \triangleq 2^{b}-1, P_{i}^{\mathcal{Q}}(t)$ is given by (12) and $\mathcal{Q} \in$ $\left\{\mathrm{H}, \mathrm{D}, \mathrm{R}_{n}, \mathrm{R}_{c}\right\}$ refers to the hybrid combiner, DC combiner, non-coherent RF combiner and coherent RF combiner, respectively. We now provide the probability of a terminal returning less than $b$ bits of feedback, i.e. $\Pi^{\mathcal{Q}}(b)=$ $\mathbb{P}\left\{P_{i}^{\mathcal{Q}}<\phi(b) d_{i}^{\alpha} \sigma^{2}\right\}$; note that $\Pi^{\mathcal{Q}}(1)$ is the idle probability. We first consider the hybrid combiner and, for the sake of tractability, we consider a hybrid combiner implementing the non-coherent RF combiner. Furthermore, we assume that the number of antenna elements are equal for all $\ell$ RF combiners which we denote by $L_{r_{0}}$. We will consider the coherent RF combiner later for the special case $L_{d}=0$ and $\ell=1$.

\footnotetext{
${ }^{2}$ The main steps of the analysis are similar for other feedback schemes; here we assume a simple feedback scheme which generalizes the work in [22].
} 
Proposition 1. The probability of returning less than $b$ bits of feedback for a terminal employing the hybrid combiner is

$$
\begin{aligned}
\Pi^{H}(b)=1-\frac{L_{r_{0}}^{-\ell}(\phi(b) \chi)^{-\frac{1}{\alpha}}}{\alpha\left(\rho^{2}-\xi^{2}\right)}\left[\sum_{k=1}^{L_{d}}(k-1) ! c_{k} \sum_{m=0}^{k-1} \frac{\Delta_{1}(m)}{m !}\right. \\
\left.+\sum_{k=1}^{\ell}(k-1) ! L_{r_{0}}^{k+\frac{1}{\alpha}} c_{k}^{\prime} \sum_{m=0}^{k-1} \frac{\Delta_{2}(m)}{m !}\right], \quad \text { (14) }
\end{aligned}
$$

where $\chi \triangleq \frac{\left(\mu V_{T}\right)^{2} \sigma^{2}}{\zeta I_{s} P_{h}}, \Delta_{i}(m) \triangleq \Gamma\left(m+\frac{1}{\alpha}, \Lambda_{i} \phi(b) \chi \xi^{2 \alpha}\right)-$ $\Gamma\left(m+\frac{1}{\alpha}, \Lambda_{i} \phi(b) \chi \rho^{2 \alpha}\right), \Lambda_{i} \in\left\{1, \frac{1}{L_{r_{0}}}\right\}, i=1,2, L_{r_{0}}>1$; $c_{k}$ and $c_{k}^{\prime}$ are given by (29), (30) and (32), (33) respectively.

Proof. See Appendix A.

From the above, we can deduce the probability of returning less than $b$ bits of feedback for a terminal employing a strictly DC or RF combiner. Specifically, by setting $\ell=0$ or $L_{r_{0}}=1$ in (14) we get the following.

Corollary 1. The probability of returning less than $b$ bits of feedback for a terminal employing the DC combiner is

$$
\begin{aligned}
\Pi^{D}(b) & =1-\frac{1}{\alpha\left(\rho^{2}-\xi^{2}\right)}(\phi(b) \chi)^{-\frac{1}{\alpha}} \sum_{m=0}^{L-1} \frac{1}{m !} \\
\times & {\left[\Gamma\left(m+\frac{1}{\alpha}, \phi(b) \chi \xi^{2 \alpha}\right)-\Gamma\left(m+\frac{1}{\alpha}, \phi(b) \chi \rho^{2 \alpha}\right)\right] . }
\end{aligned}
$$

Moreover, consider the case where the hybrid combiner employs multiple sub-array RF combiners and no single antenna element is connected directly to the DC combiner. In this case, the probability of returning less than $b$ bits of feedback can be easily deduced from (14) by setting $L_{d}=0$. Below, we provide the special case for $\ell=1$.

Corollary 2. The probability of returning less than $b$ bits of feedback for a terminal employing the non-coherent $R F$ combiner with one antenna array $(\ell=1)$ is

$$
\begin{aligned}
\Pi^{R_{n}}(b)=1- & \frac{1}{\alpha\left(\rho^{2}-\xi^{2}\right)}\left(\frac{L}{\phi(b) \psi}\right)^{\frac{1}{\alpha}} \\
& \times\left[\Gamma\left(\frac{1}{\alpha}, \phi(b) \frac{\psi}{L} \xi^{2 \alpha}\right)-\Gamma\left(\frac{1}{\alpha}, \phi(b) \frac{\psi}{L} \rho^{2 \alpha}\right)\right],
\end{aligned}
$$

where $\psi \triangleq \frac{\left(\mu V_{T}\right)^{2} \sigma^{2}}{\zeta I_{s} P_{h}}$.

Finally, we consider the coherent RF combiner for the special case $\ell=1$. We have the following proposition.

Proposition 2. The probability of returning less than $b$ bits of feedback for a terminal employing the coherent $R F$ combiner with one antenna array $(\ell=1)$ is

$$
\begin{aligned}
\Pi^{R_{c}}(b) & =1-\frac{1}{\alpha\left(\rho^{2}-\xi^{2}\right)}\left(\frac{2 \beta}{\phi(b) \psi}\right)^{\frac{1}{\alpha}} \sum_{k=0}^{L-1} \frac{1}{k !} \\
\times & {\left[\Gamma\left(k+\frac{1}{\alpha}, \frac{\phi(b) \psi \xi^{2 \alpha}}{2 \beta}\right)-\Gamma\left(k+\frac{1}{\alpha}, \frac{\phi(b) \psi \rho^{2 \alpha}}{2 \beta}\right)\right], }
\end{aligned}
$$

where $\beta=((2 L-1) ! !)^{1 / L}$.

Proof. See Appendix B.

\section{Beam Outage Probability}

In this section, we analytically derive the performance of the proposed scheme in terms of the beam outage probability. A beam is in outage when the achieved SINR is less than a target SINR $\tau$; without loss of generality, we study the outage performance of the $l$-th beam. An outage event for the $l$-th beam, occurs when no terminal returns a positive feedback for the achieved beam SINR and the link (if any) between the $\mathrm{AP}$ and the randomly selected terminal is in outage. The case where all terminals return a negative feedback is equivalent to the case where the maximum achieved beam SINR, among the active terminals, is lower than the requested threshold. We first need to derive the cumulative distribution function (CDF) of the observed beam SINR at a terminal, the CDF of the maximum beam SINR at the AP as well as the density of the terminals which return feedback (positive or negative). These are provided in the following propositions. For tractability, we first consider the case where each terminal employs the selection combiner (SC) scheme, that is $N_{c}=1$, and state the following proposition.

Proposition 3. The CDF of the observed beam SINR $\gamma$ when the terminals employ the $S C$ scheme is given by

$$
\begin{aligned}
F_{\gamma}(\tau)= & +\frac{2}{\alpha\left(\rho^{2}-\xi^{2}\right)}\left(\frac{P_{t}}{\sigma^{2} \tau}\right)^{\frac{2}{\alpha}} \sum_{m=1}^{N} \frac{(-1)^{m}}{m^{\frac{2}{\alpha}}(\tau+1)^{m(M-1)}} \\
& \times\left(\begin{array}{l}
N \\
m
\end{array}\right)\left[\Gamma\left(\frac{2}{\alpha}, \frac{\sigma^{2} \tau m \xi^{\alpha}}{P_{t}}\right)-\Gamma\left(\frac{2}{\alpha}, \frac{\sigma^{2} \tau m \rho^{\alpha}}{P_{t}}\right)\right],
\end{aligned}
$$

where $\tau$ is the target SINR threshold.

Proof. See Appendix C.

We now consider the case where the terminals employ the GSC scheme. Due to the analytical complexity of deriving the CDF of the observed SINR for this case, we assume that no inter-beam interference exists which provides a useful performance bound. One way to achieve this, is by employing antenna arrays at the AP to perform directional beamforming [32]. As a result, the antenna patterns partition the area into $M$ orthogonal sectors, each of density $\lambda / M$, and the AP transmits each beam to a pre-assigned sector. Thus, a terminal observes the SNR for its sector's beam, by combining the $N_{c}$ strongest channel paths.

Proposition 4. The CDF of the observed beam SNR $\gamma$ when the terminals employ the GSC scheme in an interference-free network is given by (19) where $\tau$ is the target SNR threshold.

Proof. See Appendix D.

Following the same reasoning as in [27], when $N_{c}=1$ and $M=1$, (19) reduces to (18).

Proposition 5. The CDF of the maximum beam SINR $\gamma_{l}^{*}$, assuming there are $n$ active terminals in the network is 


$$
\begin{aligned}
& F_{\gamma}(\tau)=\left(\begin{array}{c}
N \\
N_{c}
\end{array}\right)\left\{1-\frac{2\left(\frac{P_{t}}{\sigma^{2} \tau}\right)^{\frac{2}{\alpha}}}{\alpha\left(\rho^{2}-\xi^{2}\right)} \sum_{k=0}^{N_{c}-1} \frac{1}{k !}\left[\Gamma\left(k+\frac{2}{\alpha}, \frac{\sigma^{2} \tau \xi^{\alpha}}{P_{t}}\right)-\Gamma\left(k+\frac{2}{\alpha}, \frac{\sigma^{2} \tau \rho^{\alpha}}{P_{t}}\right)\right]+\sum_{k=1}^{N-N_{c}}\left(\begin{array}{c}
N-N_{c} \\
k
\end{array}\right)\left(\frac{N_{c}}{k}\right)^{N_{c}-1}\right. \\
& \times(-1)^{k+N_{c}-1}\left[\left(1+\frac{k}{N_{c}}\right)^{-1}\left(1-\frac{2\left(\frac{P_{t} N_{c}}{\sigma^{2} \tau\left(N_{c}+k\right)}\right)^{\frac{2}{\alpha}}}{\alpha\left(\rho^{2}-\xi^{2}\right)}\left[\Gamma\left(\frac{2}{\alpha}, \frac{\sigma^{2} \tau\left(N_{c}+k\right) \xi^{\alpha}}{P_{t} N_{c}}\right)-\Gamma\left(\frac{2}{\alpha}, \frac{\sigma^{2} \tau\left(N_{c}+k\right) \rho^{\alpha}}{P_{t} N_{c}}\right)\right]\right)\right. \\
&\left.\left.-\sum_{l=0}^{N_{c}-2}\left(-\frac{k}{N_{c}}\right)^{l}\left(1-\frac{2\left(\frac{P_{t}}{\sigma^{2} \tau}\right)^{\frac{2}{\alpha}}}{\alpha\left(\rho^{2}-\xi^{2}\right)} \sum_{m=0}^{l} \frac{1}{m !}\left[\Gamma\left(m+\frac{2}{\alpha}, \frac{\sigma^{2} \tau \xi^{\alpha}}{P_{t}}\right)-\Gamma\left(m+\frac{2}{\alpha}, \frac{\sigma^{2} \tau \rho^{\alpha}}{P_{t}}\right)\right]\right)\right]\right\} .
\end{aligned}
$$

$F_{\gamma^{*}}(\tau \mid n)=\left[F_{\gamma}(\tau)\right]^{n}$, where $F_{\gamma}(\tau)$ is given by (18) and (19) for the SC and GSC scheme, respectively.

Proof. See Appendix E.

Proposition 6. The terminals that return feedback for the l-th beam form a homogeneous $P P P \Phi_{l}^{\mathcal{Q}}$ with density

$$
\lambda^{\mathcal{Q}}=\left[1-\Pi^{\mathcal{Q}}(M)+\sum_{b=1}^{M-1} \frac{b}{M}\left(\Pi^{\mathcal{Q}}(b+1)-\Pi^{\mathcal{Q}}(b)\right)\right] \lambda,
$$

and the ones that are idle form a homogeneous PPP with density $\overline{\lambda^{\mathcal{Q}}}=\lambda-\lambda^{\mathcal{Q}}$, where $\mathcal{Q} \in\left\{H, D, R_{n}, R_{c}\right\}$.

Proof. See Appendix F.

We can now state the main results of this section. In what follows, we consider the beam outage probability for different antenna assignment scenarios: static, dynamic (distancedependent) and optimal.

\section{A. Static antenna assignment scheme}

In this scenario, the size of both the rectenna and antenna array, i.e. $L$ and $N$, at the terminals is decided a-priori. Therefore, the beam outage probability is given by the following theorem.

Theorem 1. The beam outage probability for the l-th beam and the $\mathcal{Q}$ combiner is given by

$$
\begin{aligned}
P_{\text {out }}^{\mathcal{Q}}=\exp & \left(-\lambda^{\mathcal{Q}}|\mathcal{B}|\left(1-F_{\gamma}(\tau)\right)\right) \\
\times & {\left[\exp \left(-\overline{\lambda^{\mathcal{Q}}}|\mathcal{B}|\right)\left(1-F_{\gamma}(\tau)\right)+F_{\gamma}(\tau)\right], }
\end{aligned}
$$

where $\mathcal{Q} \in\left\{H, D, R_{n}, R_{c}\right\},|\mathcal{B}|=\pi\left(\rho^{2}-\xi^{2}\right), \lambda^{\mathcal{Q}}$ and $\overline{\lambda^{\mathcal{Q}}}$ are given in Proposition 6, and $F_{\gamma}(\tau)$ is defined by (18) for the SC scheme and by (19) for the GSC scheme.

Proof. See Appendix G.

Remark 1. For $P_{t}, P_{h} \rightarrow \infty$, the beam outage probability of the SC scheme, for all rectenna architectures asymptotically converges to

$$
P_{\text {out }}^{\infty}=\exp \left(-\lambda \pi\left(\rho^{2}-\xi^{2}\right)\left(1-F_{\gamma}^{\infty}(\tau)\right)\right),
$$

where $F_{\gamma}^{\infty}(\tau)=\left[1-\frac{1}{(\tau+1)^{M-1}}\right]^{N}$.
Proof. For $P_{t}, P_{h} \rightarrow \infty$, all the terminals successfully transmit at least $M$ bits of feedback and so $\lambda^{\mathcal{Q}} \rightarrow \lambda$. Therefore, the outage probability in this case is simply $P_{\text {out }}=$ $\mathbb{E}\left\{\gamma_{l}^{*}<\tau \mid \Phi_{l}\right\}$, and the result follows.

Hence, in the high transmit power regime, the beam outage probability is independent of both the size of the rectenna array and the implemented combiner. This is expected since in this case, all terminals harvest enough energy to feed back $M$ bits, irrespective of the rectenna architecture. Furthermore, it is clear that (22) remains constant with respect to $P_{t}$ and $P_{h}$. Therefore, the outage performance asymptotically converges to an error floor which implies the diversity gain is zero. On the other hand, the outage performance of the GSC scheme at the high transmit power regime, reduces to zero due to the absence of inter-beam interference.

\section{B. Distance-based antenna assignment scheme}

We now consider a dynamic antenna assignment scenario based on an antenna switching approach [33] according to the distance of the terminal from the origin; this can be implemented with the use of dual-band antennas [34]. In particular, the coverage area is "split" into $S$ annuluses (circular rings), each of area $\pi\left(\rho_{i}^{2}-\xi_{i}^{2}\right), 1 \leq i \leq S$; note that $\rho_{S}=\rho$ is the disk's radius and $\xi_{1}=\xi$ is the exclusion zone's radius. A terminal located in the $i$-th annulus, assigns $L_{i}$ antennas for energy harvesting and $N_{i}=K-L_{i}$ antennas for data decoding. In this way, this scheme provides a potential solution in alleviating the doubly near-far problem, i.e. a terminal further away from the PB harvests less energy but requires more power to transmit the same data compared to a terminal closer to the PB [17]. Therefore, by increasing the number of rectennas with respect to the distance from the $\mathrm{PB}$, a terminal further away will potentially be able to transmit as many bits of feedback as a terminal closer to the PB. For instance, a terminal located at the edge of the coverage area, i.e. at distance $\rho$, prioritizes energy harvesting to decrease its idle probability and so increases its rectenna array. On the other hand, a terminal located at the edge of the exclusion zone, i.e. at distance $\xi$, prioritizes data decoding to increase its antenna diversity since it can power its feedback mechanism with a smaller rectenna array.

The beam outage probability of this scheme is given in the theorem below. We slightly abuse the notation used so far by generalizing our derived expressions for the $i$-th annulus, and 
thus consider the functions $F_{\gamma}\left(\tau, N_{i}, \rho_{i}, \xi_{i}\right), \Pi \mathcal{Q}\left(b, \rho_{i}, \xi_{i}, L_{i}\right)$, and $\lambda^{\mathcal{Q}}\left(\rho_{i}, \xi_{i}, L_{i}\right)$ in place of $F_{\gamma}(\tau), \Pi^{\mathcal{Q}}(b)$, and $\lambda^{\mathcal{Q}}$ respectively.

Theorem 2. The beam outage probability for the l-th beam and the $\mathcal{Q}$ combiner, $\mathcal{Q} \in\left\{R_{n}, R_{c}, D, H\right\}$, under the distancebased antenna assignment scheme is given by

$$
\begin{aligned}
P_{\text {out }}^{\mathcal{Q}}= & \exp \left(-\sum_{i=1}^{S} \lambda^{\mathcal{Q}}\left(\rho_{i}, \xi_{i}, L_{i}\right)\left|\mathcal{B}_{i}\right|\left(1-F_{\gamma}\left(\tau, N_{i}, \rho_{i}, \xi_{i}\right)\right)\right) \\
& \times \sum_{i=1}^{S} \frac{\rho_{i}^{2}-\xi_{i}^{2}}{\rho_{S}^{2}-\xi_{1}^{2}}\left(\exp \left(-\overline{\lambda^{\mathcal{Q}}}\left(\rho_{i}, \xi_{i}, L_{i}\right)\left|\mathcal{B}_{i}\right|\right)\right. \\
& \left.\times\left(1-F_{\gamma}\left(\tau, N_{i}, \rho_{i}, \xi_{i}\right)\right)+F_{\gamma}\left(\tau, N_{i}, \rho_{i}, \xi_{i}\right)\right),
\end{aligned}
$$

where $\left|\mathcal{B}_{i}\right|=\pi\left(\rho_{i}^{2}-\xi_{i}^{2}\right), 1 \leq i \leq S$.

Proof. The proof follows similarly as the one of Theorem 1. The main point here is that we need to consider each annulus separately, where the density of the terminals in the $i$ th annulus that return feedback has density $\lambda^{\mathcal{Q}}\left(\rho_{i}, \xi_{i}, L_{i}\right)$.

Remark 2. For $P_{t}, P_{h} \rightarrow \infty$, the beam outage probability of the distance-based scheme, for all rectenna architectures asymptotically converges to

$$
P_{\text {out }}^{\infty}=\exp \left(-\sum_{i=1}^{S} \lambda\left|\mathcal{B}_{i}\right|\left(1-F_{\gamma}^{\infty}\left(\tau, N_{i}\right)\right)\right),
$$

where $F_{\gamma}^{\infty}\left(\tau, N_{i}\right)=\left[1-\frac{1}{(\tau+1)^{M-1}}\right]^{N_{i}}$.

The above remark is derived similarly to Remark 1 and so we omit the proof. As expected, the distance-based scheme exhibits zero diversity gain since it follows the SC scheme.

\section{Network-wide optimal scheme}

Finally, we look at optimizing the beam outage probability of the static scenario above. Specifically, given $K$ antenna elements to choose from, we evaluate the optimal parameter $L^{*}$ for a specific combiner $\mathcal{Q}$ which minimizes the beam outage probability of the network. We call this a networkwide optimal scheme as it provides the optimal parameter $L^{*}$, common for all terminals of the network. In other words, $L^{*}$ may not be the optimal parameter for the individual performance of a certain terminal. Therefore, this scheme does not always provide the best performance. The optimization problem is formulated as follows

$$
\begin{array}{cc}
\left\{N^{*}, L^{*}\right\}= & \arg \min _{N, L} P_{\text {out }}^{\mathcal{Q}}, \\
\text { subject to } & 1 \leq N<K, \\
& 1 \leq L<K, \\
& N+L=K .
\end{array}
$$

The evaluation of these parameters is done during the initialization stage of the system and depends on the average statistics of the path-loss and fading effects. The optimal $L^{*}$ can be found by an exhaustive search over $L \in[1, K-1]$. Thus, in order to determine the optimal combination, $K-1$

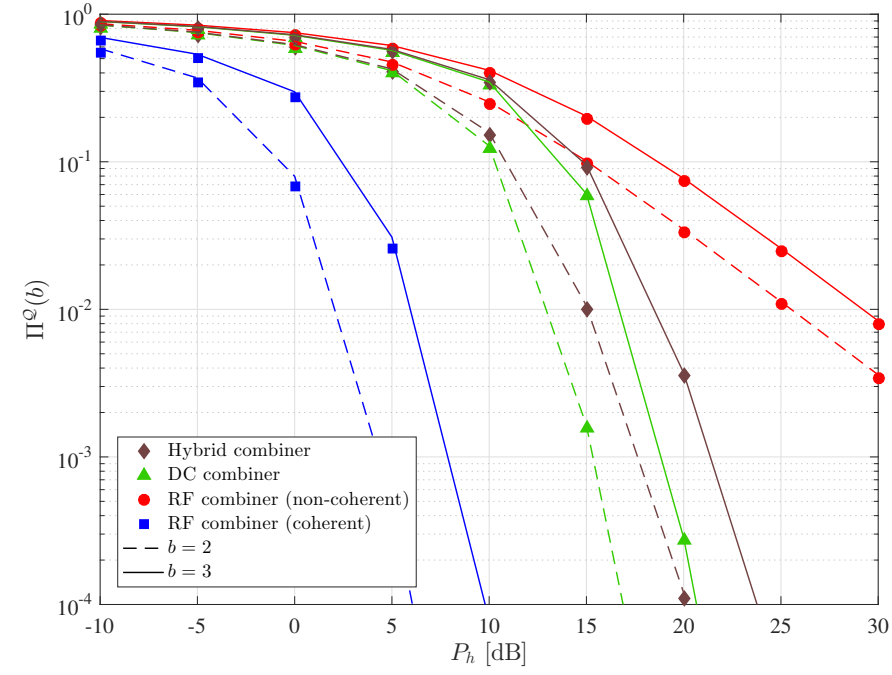

(a) $\zeta=0.9$

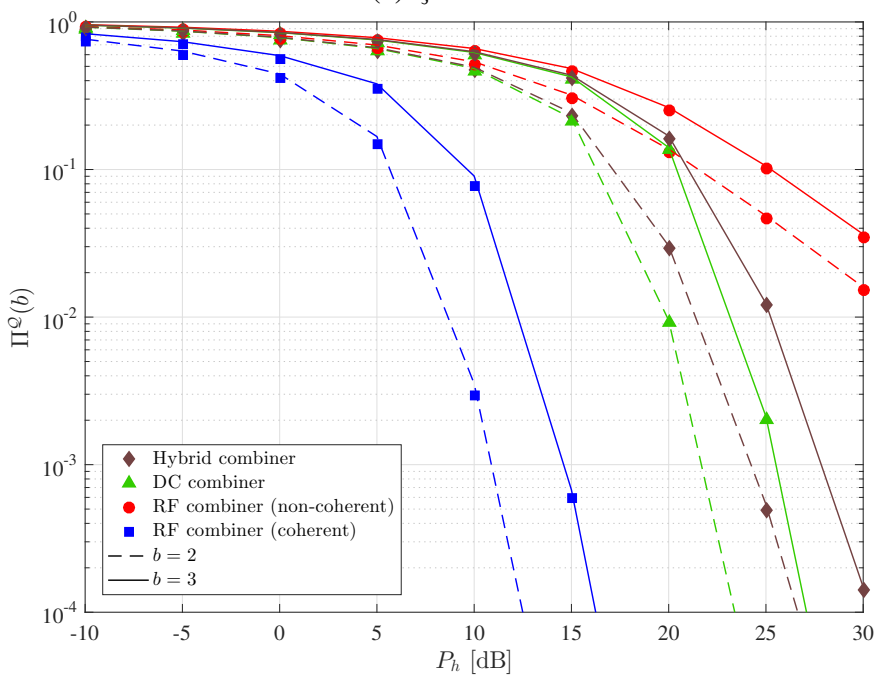

(b) $\zeta=0.2$

Fig. 3: $\Pi^{\mathcal{Q}}(b)$ versus the PB's transmit power $P_{h}$ for each rectenna array architecture; $L=8, L_{d}=4, L_{r}=4, \ell=1$.

outage probabilities need to be compared which retains a lowcomplexity. For the case $P_{t}, P_{h} \rightarrow \infty$, we have $L^{*}=1$ and so the asymptotic optimal performance is given by Remark 1 with $N^{*}=K-1$.

\section{NUMERICAL RESULTS}

We now validate and evaluate our proposed model with computer simulations. Unless otherwise stated, the simulations use the following parameters: $M=2, \lambda=0.5, \xi=2 \mathrm{~m}$, $\rho=10 \mathrm{~m}, \alpha=3, \tau=20 \mathrm{~dB}, I_{s}=1 \mathrm{~mA} \mathrm{[18],} V_{T}=28.85$ $\mathrm{mV}$ [18], $\mu=2, \zeta=0.9$ and $\sigma^{2}=-50 \mathrm{dBm}$. Furthermore, we consider that $P_{t}=P_{h}, L_{d}=L_{r}=L / 2$ and $\ell=1$. Note that these parameters have been chosen for the purpose of presenting our results and a different selection of these values will lead to the same conclusions.

Fig. 3 illustrates the probability $\Pi^{\mathcal{Q}}(b)$ of a terminal returning less than $b$ bits of feedback for all the considered rectenna architectures under two cases: $\zeta=0.9$ and $\zeta=0.2$. 


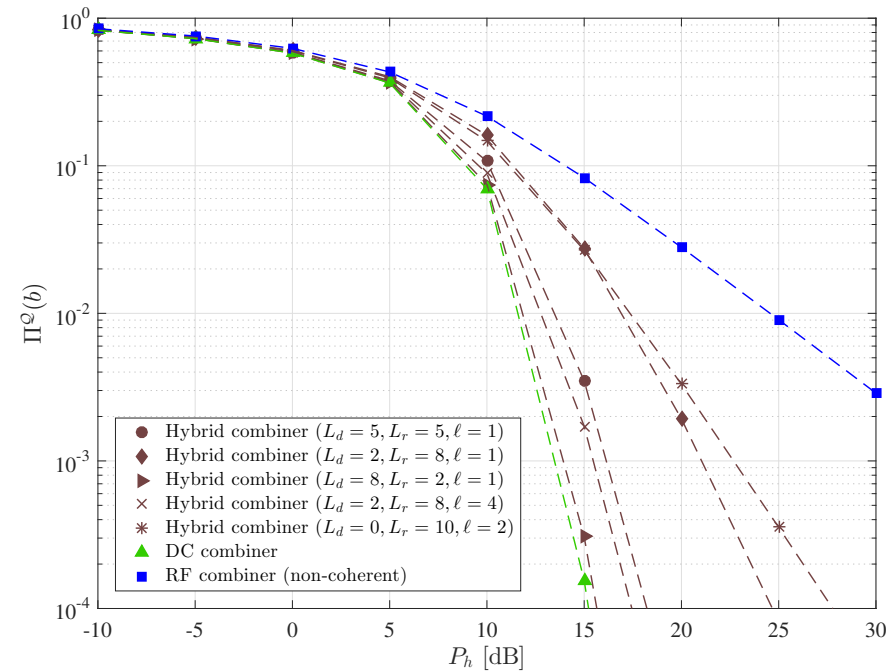

Fig. 4: $\Pi^{\mathcal{Q}}(b)$ versus the PB's transmit power $P_{h}$ for different configurations of the hybrid combiner; $L=10, b=2$ and $\lambda=0.5$.

It is obvious that the former case provides significantly better performance for all architectures which is expected since in this case the combiners experience no losses when combining the received signals. On the other hand, low combining efficiency provides lower performance. Despite this, $\Pi^{\mathcal{Q}}(b)$ decreases for high transmit power values in both cases. The figures also show the performance in terms of the requested feedback length. When the terminals are required to feed back $b=3$ bits, their performance is much lower than when $b=2$ bits, which is expected. Comparing the performance of the architectures, we can see that the best is provided by the coherent RF combiner. This confirms our earlier remark that combining the signals co-phased produces significant gains. The worst performance is provided by the non-coherent RF combiner whereas the DC combiner comes second best. Note that these observations are true when the same parameter $\zeta$ is used for all combiners. On the other hand, a more efficient non-coherent RF combiner could outperform a DC combiner. As expected, the hybrid combiner's performance lies between the performance of the DC and the non-coherent RF combiner since its rectifying resources are split between the two. Indeed, this is true for any configuration of the hybrid combiner, as can be seen in Fig. 4, where the performance of the coherent RF and DC combiner are the upper and lower bounds, respectively. The figure shows the performance of five configurations using $L=10$ rectenna elements. The main observation is that the configurations that assign more elements to the DC combiner perform better. Furthermore, a lower $\Pi^{\mathcal{Q}}(b)$ can be obtained from the configurations that implement more RF combiner sub-arrays. Finally, the theoretical results (lines) perfectly match the simulation results (markers) which validates our analysis.

Fig. 5 depicts the beam outage probability versus the transmit power for all combiner architectures with $N=4$, $L=6$, when the terminals employ the SC scheme. The proposed scheme is compared with the random beamforming

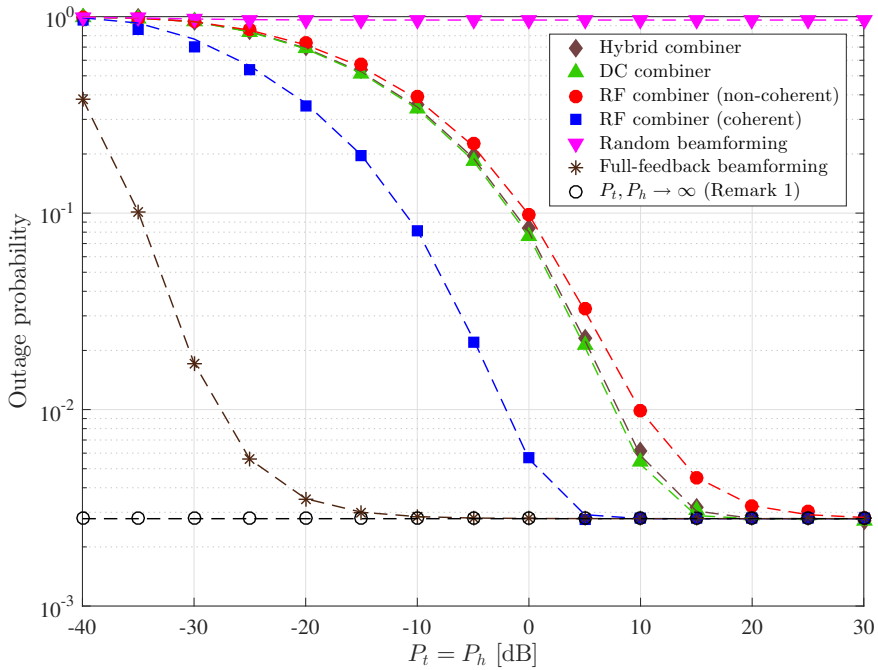

Fig. 5: Beam outage probability versus the transmit power for the SC scheme with $P_{t}=P_{h} ; N=4, L=6$ and $\lambda=0.5$.

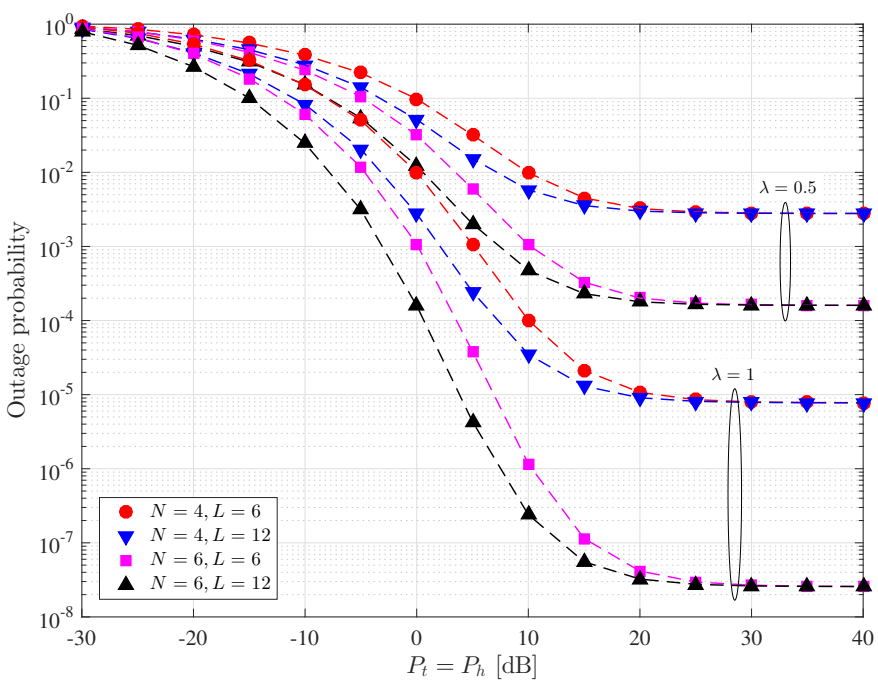

Fig. 6: Beam outage probability versus the transmit power for the SC scheme with $P_{t}=P_{h} ; N=\{4,6\}, L=\{6,12\}$ and $\lambda=\{0.5,1\}$.

(no feedback) and the full-feedback beamforming [8], i.e. when the terminals return SINR information for all beams. The first main observation is that the proposed scheme significantly outperforms the random beamforming. On the other hand, the proposed scheme does not perform as well as the full-feedback beamforming. This is expected since full feedback means that the AP has full knowledge of the terminals' SINRs. However, the performances converge to the same outage probability floor for $P_{t}, P_{h} \rightarrow \infty$. Regarding the performance of each architecture, similar observations can be deduced as before. It is obvious that the coherent RF combiner achieves a lower outage probability for low to moderate $P_{t}$ and $P_{h}$ values. At the high transmit power regime, all architectures converge to the same outage probability floor given by Remark 1 . Note that even though the hybrid combiner implements the two combiners in an equal way, it is clear from the plot that its 


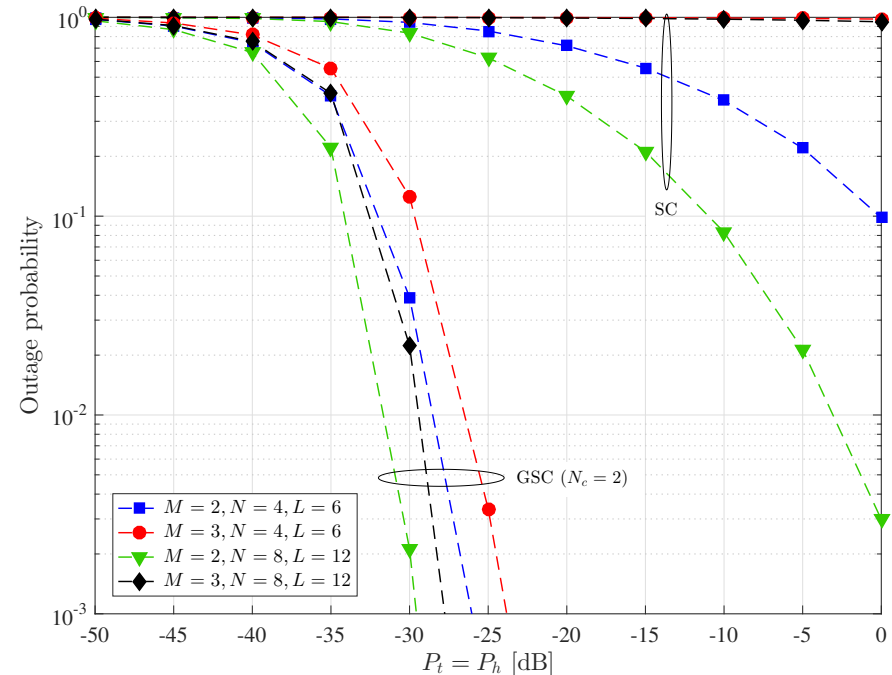

Fig. 7: Beam outage probability versus the transmit power with $P_{t}=P_{h}$ for the GSC and SC scheme using the non-coherent $\mathrm{RF}$ combiner; $M=\{2,3\}, N=\{4,8\}, L=\{6,12\}$ and $\lambda=0.5$.

performance is much closer to the DC's rather than the RF's. As before, the theoretical results (lines) perfectly match the simulation results (markers) which validates our analysis for the outage probability.

The impact of the number of antennas $N$ and the number of elements $L$ is also shown in Fig. 6. Clearly, an increase in either $N$ or $L$ improves the outage probability since the receive diversity increases with $N$ and the harvested energy increases with $L$. It is obvious from the figure, that doubling $L$ improves the outage probability for low to moderate transmit power values. For high transmit power values, the outage probability converges to an error floor regardless of $L$. On the other hand, a slight increase to the number of antennas $N$ provides substantial performance gains and further reduces the error floor. It is important to note that increasing only the size of the rectenna array does not necessarily provide great performance gains since it doesn't guarantee that the additional feedback returned to the AP will be positive. However, the receive diversity provided by an increase to $N$, increases the probability the SINR at one of the antennas achieves the required threshold. Finally, Fig. 6 also depicts the impact of the cell's density. It is clear that our proposed scheme exploits multiuser diversity, similarly to the conventional OBF scheme. Specifically, a denser network achieves better performance as more terminals will be actively requesting a specific set of beams.

In Fig. 7, we show the achieved performance of the GSC scheme with $N_{c}=2$ and compare it to the SC scheme. The results consider the employment of the non-coherent RF combiner. For a fair comparison, we consider the transmit power of the AP and $\mathrm{PB}$ in the GSC scenario for each sector to be $P_{t} / M$ and $P_{h} / M$, respectively, where $P_{t}$ and $P_{h}$ are the corresponding transmit powers in the SC scenario. It is clear that, the GSC scheme significantly outperforms the SC scheme. Moreover, the two schemes exhibit different

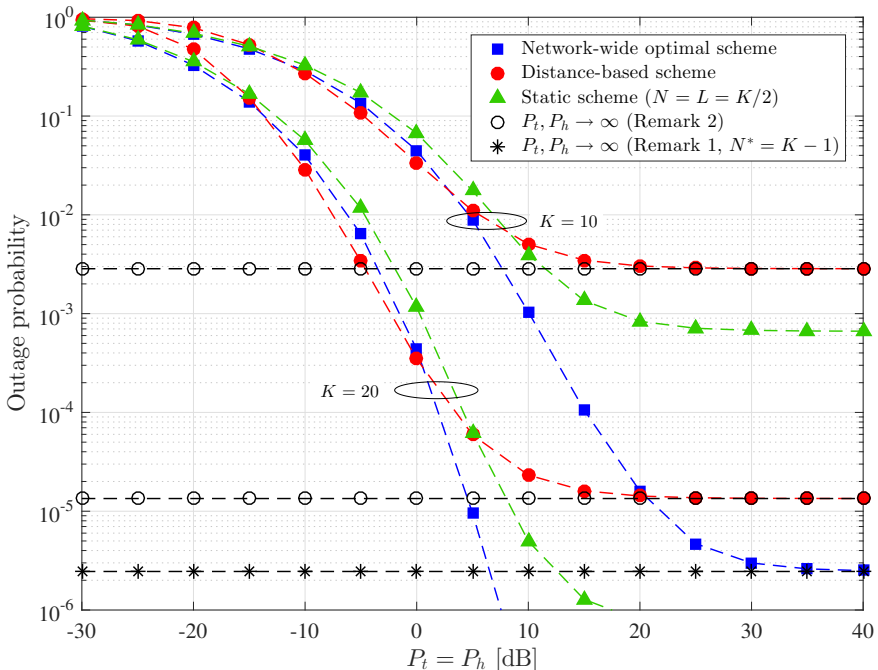

Fig. 8: Beam outage probability versus the transmit power with $P_{t}=P_{h}$ for the considered antenna assignment schemes; $M=2, S=K-1$ and $\lambda=0.5$.

performance slopes which indicate the different diversity gains and validate our remarks in Section V. An important observation is that the GSC scheme can achieve good performance even for larger values of $M$ due to the absence of interbeam interference. Furthermore, the gains obtained by the employment of the GSC scheme can be accomplished with a small value of $N_{c}$, in this case $N_{c}=2$, which keeps the complexity at low levels. An increase of $M$, decreases the performance of both schemes; the SC scheme cannot overcome the negative effects of the inter-beam interference even with large antenna and rectenna arrays, whereas the GSC scheme experiences losses in terms of multiuser diversity and transmit power.

Finally, Fig. 8 compares the considered antenna assignment schemes, namely the static SC scheme along with the distancebased and network-wide optimal schemes. The distance-based scheme is configured as follows. We consider $S=K-1$ annuluses where in the $i$-th one, $N_{i}=K-i$ and $L_{i}=i$. Hence, the lower and upper boundary of the $i$-th annulus from the origin is given by $\xi_{i}=\xi+(\rho-\xi) \frac{i-1}{K-1}$ and $\rho_{i}=\xi+(\rho-\xi) \frac{i}{K-1}$, respectively. Note that this configuration is used due to its simplicity but the distance-based scheme is general and other configurations could also be applied. As expected, the optimal scheme achieves good performance and outperforms the rest for high transmit power values. However, the distance-based antenna assignment, outperforms the optimal for moderate $P_{t}$ and $P_{h}$ values. As explained in Section V-C, the networkwide optimal scheme provides the network-wide optimal parameters $\left(L^{*}, N^{*}\right)$ to all terminals which minimizes the outage probability in the static scenario. However, this pair does not always guarantee the lowest possible outage probability, since there could be a local optimal pair $(L, N)$ for each terminal that could provide better performance. Indeed, in the distancebased assignment scheme, the terminals of each annulus are assigned a different pair of $L$ and $N$. Therefore, for moderate values of $P_{t}$ and $P_{h}$, more terminals can operate their feedback 
mechanisms compared to the optimal scheme which leads to a lower outage probability.

\section{CONCLUSION}

This paper investigated the OBF scheme in a WPCN where multi-antenna terminals harvest energy from RF signals using a rectenna array and adapt their feedback length based on the power harvested. Two fundamental rectenna architectures for the combination of the rectenna elements were investigated, namely the DC combiner and the RF combiner, as well as the hybrid DC/RF combiner; the RF combiner was considered in two different configurations, coherent and noncoherent, depending on whether the received signals were cophased. The probability of a terminal returning less than $b$ bits of feedback was derived in closed form for all of the aforementioned architectures. Furthermore, the beam outage probability was derived for the proposed OBF protocol under static and dynamic antenna assignment schemes. Our results show that the adaptation of the feedback to the harvesting efficiency of the links, improves the achieved performance and is a promising solution for the future massive network deployments such as machine-to-machine communications and sensor networks, in particular, the Internet of Things.

\section{APPENDIX A}

\section{PROOF OF PROPOSITION 1}

The hybrid combiner employs $L_{d}$ antenna elements that are connected directly to the DC combiner and $\ell$ sub-arrays of the RF combiner with $L_{r_{0}}$ antenna elements each; each subarray is connected to the DC combiner. Therefore, using (10), (12) and (13), the probability that the $i$-th terminal returns less than $b$ bits of feedback can be written as $\Pi^{\mathrm{H}}(b)=$ $\mathbb{P}\left\{Z<\phi(b) \chi d_{i}^{2 \alpha}\right\}$, where $\chi \triangleq \frac{\left(\mu V_{T}\right)^{2} \sigma^{2}}{\zeta I_{s} P_{h}}$ and $Z \triangleq X+Y$ is a generalized integer Gamma distribution; $X \triangleq \sum_{j=1}^{L_{d}}\left|g_{i, j}(t)\right|^{2}$ is a Gamma random variable with shape parameter $s_{1}=L_{d}$ and scale parameter $\Lambda_{1}=1$, and $Y \triangleq \sum_{j=1}^{\ell}\left|c_{i, j}(t)\right|^{2}$ is a Gamma random variable with shape parameter $s_{2}=\ell$ and scale parameter $\Lambda_{2}=1 / L_{r_{0}}$. We deal with the case where the scale parameters of the two random variables are different, i.e. $L_{r_{0}}>1$. The CDF of the random variable $Z$ is given by [35],

$$
F_{Z}(x)=1-\Lambda_{1}^{s_{1}} \Lambda_{2}^{s_{2}}\left(P_{X}(x)+P_{Y}(x)\right),
$$

where

$$
P_{X}(x)=\exp \left(-\Lambda_{1} x\right) \sum_{k=1}^{s_{1}}(k-1) ! c_{k} \sum_{m=0}^{k-1} \frac{1}{m !} \frac{x^{m}}{\Lambda_{1}^{k-m}},
$$

with

$$
\begin{aligned}
& c_{s_{1}}=\frac{\left(\Lambda_{2}-\Lambda_{1}\right)^{-s_{2}}}{\left(s_{1}-1\right) !} \\
& c_{s_{1}-n}=\frac{s_{2}}{n} \sum_{j=1}^{n} \frac{\left(s_{1}-n+j-1\right) !}{\left(s_{1}-n-1\right) !\left(\Lambda_{1}-\Lambda_{2}\right)^{j}} c_{s_{1}-(n-j)},
\end{aligned}
$$

where $n=1, \ldots, s_{1}-1$, and

$$
P_{Y}(x)=\exp \left(-\Lambda_{2} x\right) \sum_{k=1}^{s_{2}}(k-1) ! c_{k}^{\prime} \sum_{m=0}^{k-1} \frac{1}{m !} \frac{x^{m}}{\Lambda_{2}^{k-m}}
$$

with

$$
\begin{aligned}
& c_{s_{2}}^{\prime}=\frac{\left(\Lambda_{1}-\Lambda_{2}\right)^{-s_{1}}}{\left(s_{2}-1\right) !}, \\
& c_{s_{2}-n}^{\prime}=\frac{s_{1}}{n} \sum_{j=1}^{n} \frac{\left(s_{2}-n+j-1\right) !}{\left(s_{2}-n-1\right) !\left(\Lambda_{2}-\Lambda_{1}\right)^{j}} c_{s_{2}-(n-j)}^{\prime},
\end{aligned}
$$

where $n=1, \ldots, s_{2}-1$. We can now evaluate $\Pi^{\mathrm{H}}(b)$ as follows

$$
\begin{aligned}
& \Pi^{\mathrm{H}}(b)=\mathbb{E}\left\{1-\Lambda_{1}^{s_{1}} \Lambda_{2}^{s_{2}}\left(P_{X}\left(\phi(b) \chi d_{i}^{2 \alpha}\right)+P_{Y}\left(\phi(b) \chi d_{i}^{2 \alpha}\right)\right)\right\} \\
& =1-\Lambda_{1}^{s_{1}} \Lambda_{2}^{s_{2}} \int_{0}^{2 \pi} \int_{\xi}^{\rho}\left[\exp \left(-\Lambda_{1} \phi(b) \chi r^{2 \alpha}\right)\right. \\
& \times \sum_{k=1}^{s_{1}}(k-1) ! c_{k} \sum_{m=0}^{k-1} \frac{\left(\phi(b) \chi r^{2 \alpha}\right)^{m}}{m ! \Lambda_{1}^{k-m}}+\exp \left(-\Lambda_{2} \phi(b) \chi r^{2 \alpha}\right) \\
& \left.\times \sum_{k=1}^{s_{2}}(k-1) ! c_{k}^{\prime} \sum_{m=0}^{k-1} \frac{\left(\phi(b) \chi r^{2 \alpha}\right)^{m}}{m ! \Lambda_{2}^{k-m}}\right] f_{d}(r) r d r d \theta \\
& =1-\frac{2 \Lambda_{1}^{s_{1}} \Lambda_{2}^{s_{2}}}{\rho^{2}-\xi^{2}}\left[\sum_{k=1}^{s_{1}}(k-1) ! c_{k} \sum_{m=0}^{k-1} \frac{1}{m !} \frac{(\phi(b) \chi)^{m}}{\Lambda_{1}^{k-m}} \mathcal{I}_{1}\right. \\
& \left.+\sum_{k=1}^{s_{2}}(k-1) ! c_{k}^{\prime} \sum_{m=0}^{k-1} \frac{1}{m !} \frac{(\phi(b) \chi)^{m}}{\Lambda_{2}^{k-m}} \mathcal{I}_{2}\right],
\end{aligned}
$$

where

$$
\begin{aligned}
\mathcal{I}_{i} & =\int_{\xi}^{\rho} \exp \left(-\Lambda_{i} \phi(b) \chi r^{2 \alpha}\right) r^{2 \alpha m+1} d r \\
& =\frac{\Gamma\left(m+\frac{1}{\alpha}, \Lambda_{i} \phi(b) \chi \xi^{2 \alpha}\right)-\Gamma\left(m+\frac{1}{\alpha}, \Lambda_{i} \phi(b) \chi \rho^{2 \alpha}\right)}{2 \alpha\left(\Lambda_{i} \phi(b) \chi\right)^{m+\frac{1}{\alpha}}},
\end{aligned}
$$

with $i=1,2$ and follows from [23, 3.381]. By replacing the values for the shape and scale parameters, after some algebraic manipulations, we get (14).

\section{APPENDIX B}

\section{ProOF OF PROPOSITION 2}

From (10), the output DC current of the coherent RF combiner with $\ell=1$ is

$$
I_{i}^{\mathrm{DC}}(t)=\frac{I_{s} P_{h} d_{i}^{-\alpha}}{\left(\mu V_{T}\right)^{2}}\left(\sum_{j=1}^{L}\left|g_{i, j, k}(t)\right|\right)^{2} .
$$

Hence, the probability that the $i$-th terminal returns less than $b$ bits of feedback is $\Pi^{\mathrm{R}_{c}}(b)=\mathbb{P}\left\{Z<\sqrt{\phi(b) \psi} d_{i}^{\alpha}\right\}$, where $\psi \triangleq \frac{\left(\mu V_{T}\right)^{2} \sigma^{2}}{\zeta I_{s} P_{h}}$ and $Z \triangleq \sum_{j=1}^{L}\left|g_{i, j}(t)\right|$ is the sum of $L$ independent and identical Rayleigh distributed random variables with unit scale parameter and its CDF is approximated by [36]

$$
F_{Z}(x)=1-\exp \left(-\frac{x^{2}}{2 \beta}\right) \sum_{k=0}^{L-1} \frac{1}{k !}\left(\frac{x^{2}}{2 \beta}\right)^{k},
$$

with $\beta=((2 L-1) ! !)^{1 / L}$. Therefore, we have

$$
\mathbb{P}\left\{Z<\sqrt{\phi(b) \psi} d_{i}^{\alpha}\right\}=\mathbb{E}\left\{1-\exp \left(-\frac{\left(\sqrt{\phi(b) \psi} d_{i}^{\alpha}\right)^{2}}{2 \beta}\right)\right.
$$




$$
\begin{gathered}
\left.\times \sum_{k=0}^{L-1} \frac{1}{k !}\left(\frac{\left(\sqrt{\phi(b) \psi} d_{i}^{\alpha}\right)^{2}}{2 \beta}\right)^{k}\right\} \\
=1-\int_{0}^{2 \pi} \int_{\xi}^{\rho} f_{d}(r) r \exp \left(-\frac{\phi(b) \psi r^{2 \alpha}}{2 \beta}\right) \\
\times \sum_{k=0}^{L-1} \frac{1}{k !}\left(\frac{\phi(b) \psi r^{2 \alpha}}{2 \beta}\right)^{k} d r \\
=1-\frac{2}{\rho^{2}-\xi^{2}} \sum_{k=0}^{L-1} \frac{1}{k !}\left(\frac{\phi(b) \psi}{2 \beta}\right)^{k} \\
\times \int_{\xi}^{\rho} r^{2 \alpha k+1} \exp \left(-\frac{\phi(b) \psi r^{2 \alpha}}{2 \beta}\right) d r .
\end{gathered}
$$

The result then follows from $[23,3.381]$.

\section{APPENDIX C}

\section{PROOF OF PROPOSITION 3}

The achieved SINR for the $l$-th beam at the $i$-th terminal is given by $\gamma_{i, l}=\max _{1 \leq j \leq N} \gamma_{i, j, l}$. The CDF of the observed beam SINR is written as [8]

$$
F_{\gamma}\left(\tau \mid d_{i}=d\right)=\left[1-\frac{\exp \left(-\frac{\tau d^{\alpha} \sigma^{2}}{P_{t}}\right)}{(\tau+1)^{M-1}}\right]^{N},
$$

for a given path-loss value $d$ and by taking expectation over $d$, it can be evaluated as

$$
F_{\gamma}(\tau)=\int_{0}^{2 \pi} \int_{\xi}^{\rho}\left[1-\frac{\exp \left(-\frac{\tau r^{\alpha} \sigma^{2}}{P_{t}}\right)}{(\tau+1)^{M-1}}\right]^{N} f_{d}(r) r d r d \theta
$$

Then, the final expression can be easily obtained by using the binomial theorem as well as the expressions in [23, 3.381].

\section{APPENDIX D}

\section{PROOF OF PROPOSITION 4}

In this case, the achieved SNR for the $l$-th beam at the $i$-th terminal is given by (4). The CDF of the observed beam SNR for a given path-loss value $d$ is written as [27]

$$
\begin{aligned}
& F_{\gamma}(\tau \mid d)=\left(\begin{array}{c}
N \\
N_{c}
\end{array}\right)\{1-\underbrace{\exp \left(-\frac{\tau d^{\alpha} \sigma^{2}}{P_{t}}\right) \sum_{k=0}^{N_{c}-1} \frac{1}{k !}\left(\frac{\tau d^{\alpha} \sigma^{2}}{P_{t}}\right)^{k}}_{\mathcal{F}_{1}} \\
& +\sum_{k=1}^{N-N_{c}}\left(\begin{array}{c}
N-N_{c} \\
k
\end{array}\right)\left(\frac{N_{c}}{k}\right)^{N_{c}-1}(-1)^{k+N_{c}-1}\left[\left(1+\frac{k}{N_{c}}\right)^{-1}\right. \\
& \times(1-\underbrace{\exp \left(-\frac{\tau d^{\alpha} \sigma^{2}}{P_{t}}\left(1+\frac{k}{N_{c}}\right)\right)}_{\mathcal{F}_{2}})-\sum_{l=0}^{N_{c}-2}\left(-\frac{k}{N_{c}}\right)^{l} \\
& \left.\left.\times(1-\underbrace{\exp \left(-\frac{\tau d^{\alpha} \sigma^{2}}{P_{t}}\right) \sum_{m=0}^{l} \frac{1}{m !}\left(\frac{\tau d^{\alpha} \sigma^{2}}{P_{t}}\right)^{m}}_{\mathcal{F}_{3}})\right]\right\},
\end{aligned}
$$

where by taking expectation over $d$, the expressions $\mathcal{F}_{1}, \mathcal{F}_{2}$ and $\mathcal{F}_{3}$ can be easily evaluated as above by using [23, 3.381] and the results follows.

\section{APPENDIX E \\ PROOF OF PROPOSITION 5}

By assuming that $n$ terminals return feedback, the CDF of the maximum beam SINR is written as $\gamma_{l}^{*}=\max _{1 \leq i \leq n} \gamma_{i, l}$. By using higher order statistics and conditioning on both $n$ and the path-loss values, the CDF can be evaluated as $F_{\gamma^{*}}\left(\tau \mid n, d_{1}, \ldots, d_{n}\right)=\prod_{i=1}^{n} F_{\gamma}\left(\tau \mid d_{i}\right)$. Then,

$$
\begin{aligned}
F_{\gamma^{*}}(\tau \mid n) & =\left[\int_{0}^{2 \pi} \int_{\xi}^{\rho} F_{\gamma}(\tau \mid r) f_{d}(r) d r d \theta\right]^{n} \\
& =\left[F_{\gamma}(\tau)\right]^{n},
\end{aligned}
$$

which follows by the expectation over the path-loss values.

\section{APPENDIX F}

\section{ProOF OF PROPOSITION 6}

The probability that a terminal employing the $\mathcal{Q}$ combiner can return $b$ bits of feedback is $\mathbb{P}\left\{b \leq C_{\mathcal{Q}}<b+1\right\}$, where $C_{\mathcal{Q}}$ is given by (13). As the beams are assigned randomly, by considering all $M$ ! possible assignments, the probability the $l$-th beam is one of the first $b$ beams in an assignment is $\frac{b(M-1) !}{M !}=\frac{b}{M}$. Therefore, by the thinning theorem [24], the density of the terminals returning $b$ bits of feedback (containing feedback for the $l$-th beam) is

$$
\begin{aligned}
\lambda_{b}^{\mathcal{Q}} & =\frac{b}{M} \mathbb{P}\left\{b \leq C_{\mathcal{Q}}<b+1\right\} \lambda \\
& =\frac{b}{M}\left(\Pi^{\mathcal{Q}}(b+1)-\Pi^{\mathcal{Q}}(b)\right) \lambda,
\end{aligned}
$$

for $1 \leq b \leq M-1$. If $b \geq M$, then the density is given by $\left(1-\Pi^{\mathcal{Q}}(M)\right) \lambda$. The result follows by considering the sum over all values of $b$.

\section{APPENDIX G PROOF OF THEOREM 1}

Based on the proposed protocol, the $l$-th beam is in outage when all acquired feedback is negative and the link with the randomly selected terminal (if any) does not achieve the required SINR threshold. The former is equivalent to the case where the maximum beam SINR for the $l$-th beam, among the active terminals, is lower than the required threshold. In mathematical terms, the beam outage probability for the $l$-th beam and the $\mathcal{Q}$ combiner can be expressed as

$$
P_{\text {out }}^{\mathcal{Q}}=\mathbb{P}\left\{\gamma_{l}^{*}<\tau \mid \Phi_{l}^{\mathcal{Q}}\right\} \mathbb{P}\left\{\gamma_{l}<\tau \mid \overline{\Phi_{l}^{\mathcal{Q}}}\right\},
$$

where $\mathbb{P}\left\{\gamma_{l}^{*}<\tau \mid \Phi_{l}^{\mathcal{Q}}\right\}$ is the CDF of the maximum beam SINR and $\mathbb{P}\left\{\gamma_{l}<\tau \mid \overline{\Phi_{l}^{\mathcal{Q}}}\right\}$ is the CDF of the randomly selected link's SINR. Assuming $n$ terminals return feedback to the AP

$$
\begin{aligned}
\mathbb{P}\left\{\gamma_{l}^{*}<\tau \mid \Phi_{l}^{\mathcal{Q}}\right\} & =\sum_{n=0}^{\infty} \mathbb{P}\left\{\gamma_{l}^{*}<\tau \mid n\right\} \mathbb{P}\left\{N_{\Phi_{l}^{\mathcal{Q}}}(\mathcal{B})=n\right\} \\
& =\sum_{n=0}^{\infty} \frac{\exp \left(-\lambda^{\mathcal{Q}}|\mathcal{B}|\right)\left(\lambda^{\mathcal{Q}}|\mathcal{B}| F_{\gamma}(\tau)\right)^{n}}{n !} \\
& =\exp \left(-\lambda^{\mathcal{Q}}|\mathcal{B}|\left(1-F_{\gamma}(\tau)\right)\right)
\end{aligned}
$$


where $\mathbb{P}\left\{\gamma_{l}^{*}<\tau \mid n\right\}=\left[F_{\gamma}(x)\right]^{n}$ follows from Proposition 5 and (42) follows from the power series representation of the exponential function, $\sum_{n=0}^{\infty} \frac{x^{n}}{n !}=\exp (x)$. Also, we have

$$
\begin{aligned}
\mathbb{P}\left\{\gamma_{l}<\tau \mid \overline{\Phi_{l}^{\mathcal{Q}}}\right\}= & \mathbb{P}\left\{N_{\overline{\Phi_{l}^{\mathcal{Q}}}}(\mathcal{B})=0\right\} \\
& +\mathbb{P}\left\{N_{\overline{\Phi_{l}^{\mathcal{Q}}}}(\mathcal{B})>0\right\} \mathbb{P}\left\{\gamma_{l}<\tau\right\},
\end{aligned}
$$

with

$$
\mathbb{P}\left\{N_{\overline{\Phi_{l}^{\mathcal{Q}}}}(\mathcal{B})=0\right\}=\exp \left(-\overline{\lambda^{\mathcal{Q}}}|\mathcal{B}|\right)
$$

where $\overline{\Phi_{l}^{\mathcal{Q}}}$ denotes the complementary homogeneous PPP with density $\overline{\lambda^{\mathcal{Q}}}=\lambda-\lambda^{\mathcal{Q}}$ and $|\mathcal{B}|=\pi\left(\rho^{2}-\xi^{2}\right)$. By substituting (42), (43) and (44) into (41), the theorem is proven.

\section{REFERENCES}

[1] C. Psomas and I. Krikidis, "Adaptive variable-length feedback using wireless power transfer for opportunistic beamforming," in Proc. IEEE Int. Conf. Commun., Kuala Lumpur, Malaysia, May 2016.

[2] D. Tse and P. Viswanath, Fundamentals of Wireless Communication. Cambridge University Press, 2005.

[3] C. E. Shannon, "Channels with side information at the transmitter," $I B M$ Journal Research and Dev., vol. 2, no. 4, pp. 289-293, Oct. 1958.

[4] IEEE 802.11ac-2013, IEEE Standard for information technology Telecommunications and information exchange between systems - Local and metropolitan area networks - Specific requirements. Part 11: Wireless LAN Medium Access Control (MAC) and Physical Layer (PHY) Specifications. Amendment 4: Enhancements for very high throughput for operation in bands below $6 \mathrm{GHz}$. IEEE, Inc., Dec. 2013.

[5] P. Viswanath, D. N. C. Tse, and R. Laroia, "Opportunistic beamforming using dumb antennas," IEEE Trans. Inf. Theory, vol. 48, no. 6, pp. 12771294, June 2002.

[6] M. Sharif and B. Hassibi, "On the capacity of MIMO broadcast channels with partial side information," IEEE Trans. Inf. Theory, vol. 51, no. 2, pp. 506-522, Feb. 2005.

[7] A. Bayesteh, and A. K. Khandani, "Asymptotic analysis of the amount of CSI feedback in MIMO broadcast channels," IEEE Trans. Inf. Theory, vol. 58, no. 3, pp. 1612-1629, Mar. 2012.

[8] M.-O. Pun, V. Koivunen, and H. V. Poor, "Performance analysis of joint opportunistic scheduling and receiver design for MIMO-SDMA downlink systems," IEEE Trans. Commun., vol. 59, no. 1, pp. 268-280, Jan. 2011.

[9] T. Samarasinghe, H. Inaltekin, and J. S. Evans, "Modeling and analysis of opportunistic beamforming for poisson wireless networks," IEEE Trans. Wireless Commun., vol. 15, no. 5, pp. 3732-3745, May 2016.

[10] Z. Ding and H. V. Poor, "Design of massive-MIMO-NOMA with limited feedback," IEEE Signal Proc. Lett., vol. 23, no. 5, pp. 629-633, May 2016.

[11] D. J. Love, R. W. Heath, Jr., V. K. N. Lau, D. Gesbert, B. D. Rao, and M. Andrews, "An overview of limited feedback in wireless communication systems," IEEE J. Select. Areas Commun., vol. 26, no. 8, pp. 1341-1365, Oct. 2008.

[12] J. Diaz, O. Simeone, and Y. Bar-Ness, "How many bits of feedback is multiuser diversity worth in MIMO downlink?," in Proc. IEEE Symp. Spread Spec. Tech. Appl., Manaus, Brazil, Aug. 2006, pp. 505-509.

[13] S. Sanayei and A. Nosratinia, "Opportunistic beamforming with limited feedback," IEEE Trans. Wireless Commun., vol. 6, no. 8, pp. 2765-2771, Aug. 2007.

[14] Z. Ding, C. Zhong, D. W. K. Ng, M. Peng, H. A. Suraweera, R. Schober, and H. V. Poor, "Application of smart antenna technologies in simultaneous wireless information and power transfer," IEEE Commun. Mag., vol. 53, no. 4, pp. 86-93, Apr. 2015.

[15] N. Shinohara and H. Matsumoto, "Experimental study of large rectenna array for microwave energy transmission," IEEE Trans. Microw. Theory Techn., vol. 46, no. 3, pp. 261-268, Mar. 1998.

[16] R. Zhang and C. K. Ho, "MIMO broadcast for simultaneous wireless information and power transfer," IEEE Trans. Wireless Commun., vol. 12, no. 5, pp. 1989-2001, May 2013.

[17] $\mathrm{H}$. Ju and R. Zhang, "Throughput maximization in wireless powered communication networks," IEEE Trans. Wireless Commun., vol. 13, no. 1, pp. 418-428, Jan. 2014.
[18] M. Xia and S. Aissa, "On the efficiency of far-field wireless power transfer," IEEE Trans. Sign. Proc., vol. 63, no. 11, pp. 2835-2847, Mar. 2015.

[19] G. Oliveri, L. Poli, and A. Massa, "Maximum efficiency beam synthesis of radiating planar arrays for wireless power transmission," IEEE Trans. Antennas Propag., pp. 2490-2499, vol. 61, no. 5, May 2013.

[20] A. Massa, G. Oliveri, F. Viani, and P. Rocca, "Array designs for longdistance wireless power transmission: State-of-the-art and innovative solutions," IEEE Proc., vol. 101, no. 6, pp. 1464-1481, Mar. 2013.

[21] U. Olgun, C.-C. Chen, and J. L. Volakis, "Investigation of rectenna array configurations for enhanced RF power harvesting," IEEE Ant. Wireless Prop. Lett., vol. 10, pp. 262-265, Apr. 2011.

[22] I. Krikidis, "Opportunistic beamforming with wireless powered 1-bit feedback through rectenna array," IEEE Signal Proc. Lett., vol. 22, no. 11 , pp. 2054-2058, Nov. 2015.

[23] I. S. Gradshteyn and I. M. Ryzhik, Table of Integrals, Series, and Products. Elsevier, 2007.

[24] M. Haenggi, Stochastic Geometry for Wireless Networks. Cambridge University Press, 2013.

[25] IEEE Std C95.1-2005, IEEE standard for safety levels with respect to human exposure to radio frequency electromagnetic fields, $3 \mathrm{kHz}$ to 300 GHz. IEEE, Inc., Oct. 2005.

[26] C.-F. Liu and C.-H. Lee, "Information and power transfer under MISO channel with finite-rate feedback," in Proc. IEEE Global Commun. Conf., Atlanta, GA, Dec. 2013, pp. 2497-2501.

[27] M. -S. Alouini and M. K. Simon, "An MGF-based performance analysis of generalized selection combining over Rayleigh fading channels," IEEE Trans. Commun., vol. 48, no. 3, pp. 401-415, Mar. 2000.

[28] Y. Zeng, B, Clerckx, and R. Zhang, "Communications and signals design for wireless power transfer," IEEE Trans. Commun., vol. 65, no. 5, pp. 2264-2290, May 2017.

[29] F. Meng, K. Ma, K. S. Yeo, and S. Xu, "A 57-to-64-GHz 0.094-mm² 5-bit passive phase shifter in 65-nm CMOS," IEEE Trans. Very Large Scale Integr. (VLSI) Syst., vol. 24, no. 5, pp. 1917-1925, May 2016.

[30] Y.-H. Lin, and H. Wang, "A low phase and gain error passive phase shifter in $90 \mathrm{~nm}$ CMOS for $60 \mathrm{GHz}$ phase array system application," in Proc. IEEE MTT-S Int. Microw. Symp., San Francisco, CA, May 2016.

[31] H. Liang, C. Zhong, X. Chen, H. A. Suraweera, and Zhaoyang Zhang, "Wireless powered dual-hop multi-antenna relaying systems: Impact of CSI and antenna correlation," IEEE Trans. Wireless Commun., vol. 16, no. 4, Apr. 2017

[32] D. Torrieri, S. Talarico, and M. C. Valenti, "Analysis of a frequencyhopping millimeter-wave cellular uplink," IEEE Trans. Wireless Commun., vol. 15, no. 10, pp. 7089-7098, Oct. 2016.

[33] I. Krikidis, S. Sasaki, S. Timotheou, and Z. Ding, "A low complexity antenna switching for joint wireless information and energy transfer in MIMO relay channels," IEEE Trans. Commun., vol.62, no. 5, pp.15771587, May 2014

[34] A. Costanzo, D. Masotti, M. Fantuzzi, and M. Del Prete, "Co-design strategies for energy-efficient UWB and UHF wireless systems," IEEE Trans. Microw. Theory Techn., vol. 65, no. 5, pp. 1852-1863, May 2017.

[35] C. A. Coelho, "The generalized integer Gamma distribution - A basis for distributions in multivariate statistics," J. Multivariate Anal., vol. 64, no. 1 , pp. 86-102, 1998.

[36] J. Hu and N. C. Beaulieu, "Accurate simple closed-form approximations to Rayleigh sum distributions and densities," IEEE Commun. Lett., vol. 9, no. 2, pp. 109-111, Feb. 2005.

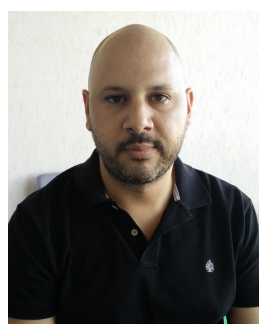

Constantinos Psomas (M'15) received a B.Sc. in Computer Science and Mathematics from Royal Holloway, University of London, UK in 2007, an M.Sc. in Applicable Mathematics from the London School of Economics, UK in 2008, and a Ph.D. in Mathematics from The Open University, UK in 2011. He is currently a Postdoctoral Researcher at the KIOS Research and Innovation Center of Excellence of the University of Cyprus. From October 2011 to March 2014, he was a Postdoctoral Research Fellow at the Department of Electrical Engineering, Computer Engineering and Informatics of the Cyprus University of Technology. His current research interests include full-duplex radio, wireless powered communications and cooperative networks. 


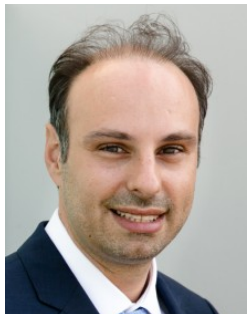

Ioannis Krikidis (S'03-M'07-SM'12) received the diploma in Computer Engineering from the Computer Engineering and Informatics Department (CEID) of the University of Patras, Greece, in 2000, and the M.Sc and Ph.D degrees from Ecole Nationale Supérieure des Télécommunications (ENST), Paris, France, in 2001 and 2005, respectively, all in electrical engineering. From 2006 to 2007 he worked, as a Post-Doctoral researcher, with ENST, Paris, France, and from 2007 to 2010 he was a Research Fellow in the School of Engineering and Electronics at the University of Edinburgh, Edinburgh, UK. He has held also research positions at the Department of Electrical Engineering, University of Notre Dame; the Department of Electrical and Computer Engineering, University of Maryland; the Interdisciplinary Centre for Security, Reliability and Trust, University of Luxembourg; and the Department of Electrical and Electronic Engineering, Niigata University, Japan. He is currently an Assistant Professor at the Department of Electrical and Computer Engineering, University of Cyprus, Nicosia, Cyprus. His current research interests include communication theory, wireless communications, cooperative networks, cognitive radio and wireless powered communications.

Dr. Krikidis serves as an Associate Editor for IEEE TRANSACTIONS ON COMMUNICATIONS, TRANSACTIONS ON GREEN COMMUNICATIONS AND NETWORKING, and IEEE WIRELESS COMMUNICATIONS LETTERS. He was the Technical Program Co-Chair for the IEEE International Symposium on Signal Processing and Information Technology 2013 as well as the Lead Guest Editor of the Special Issue on Exploiting interference towards Energy Efficient and Secure Wireless Communications, IEEE JOURNAL OF SELECTED TOPICS IN SIGNAL PROCESSING, December 2016. He was the recipient of the Research Award Young Researcher from the Research Promotion Foundation, Cyprus, in 2013, as well as the recipient of the IEEE ComSoc Best Young Professional Award in Academia in 2016. 